\title{
Therapeutic Effect of Mirogabalin on Peripheral Neuropathic Pain due to Lumbar Spine Disease
}

\author{
Kyongsong Kim ${ }^{1,2}$, Toyohiko Isu ${ }^{3}$, Rinko Kokubo ${ }^{1,4}$, Naotaka Iwamoto ${ }^{5,6}$, \\ Daijiro Morimoto ${ }^{5}$, Masaaki Kawauchi ${ }^{2}$, Akio Morita $^{5}$ \\ ${ }^{1}$ Department of Neurological Surgery, Chiba Hokuso Hospital, Nippon Medical School, Inzai, Japan \\ ${ }^{2}$ Department of Neurosurgery, Chiba Shintoshi Rurban Clinic, Inzai, Japan \\ ${ }^{3}$ Department of Neurosurgery, Kushiro Rosai Hospital, Kushiro, Japan \\ ${ }^{4}$ Department of Neurosurgery, Katsutadai Hospital, Yachiyo, Japan \\ ${ }^{5}$ Department of Neurological Surgery, Nippon Medical School, Bunkyo, Japan \\ ${ }^{6}$ Department of Neurosurgery, Tokyo-Hikifune Hospital, Sumida, Japan
}

Study Design: Retrospective study.

Purpose: This study aims to evaluate the effectiveness of mirogabalin in treatment of peripheral neuropathic pain due to lumbar spine disease.

Overview of Literature: Mirogabalin is a novel selective ligand for the $\alpha 2 \delta$ subunit of voltage-gated Ca channels.

Methods: Between April and December 2019, we used mirogabalin to treat 60 consecutive patients (mean age, 67.6 years) with leg symptoms due to lumbar disease. The treatment outcome after 8 weeks of mirogabalin therapy was evaluated by comparing the preand post-administration Numerical Rating Scale (NRS) for leg symptoms and sleep disturbance, the NRS and Roland-Morris Disability Questionnaire for low back pain (LBP), and the quality of life (QOL) score (based on EuroQol five-dimension five-level scale).

Results: Mirogabalin treatment was stopped at less than eight weeks in eight patients. The remaining 52 patients for evaluation were divided as group 1 (17 patients who presented with leg symptoms that lasted for less than 3 months) and group 2 (35 patients with leg symptoms that lasted longer than 3 months). The leg symptoms and LBP in both groups significantly improved at 4 and 8 weeks of treatment, and sleep disturbance and $\mathrm{OOL}$ were improved at 8 weeks as well. Compared to group 2, the pretreatment leg symptoms and $\mathrm{OOL}$ were significantly worse in group 1, and their improvement after 8 weeks of mirogabalin treatment was significantly better $(p<0.05)$. Of the 60 original patients, 17 suffered adverse effects, which were mild in 16 patients and required treatment cessation due to excessive weight gain in one patient.

Conclusions: We have validated the effect of mirogabalin on neuropathic pain due to lumbar spine disease, which has effectively addressed the associated leg symptoms, LBP, and sleep disturbance.

Keywords: Mirogabalin; Leg symptom; Low back pain; Lumbar spine disease; Neuralgia

Received Mar 31, 2020; Revised Apr 22, 2020; Accepted Apr 23, 2020

Corresponding author: Kyongsong Kim

Department of Neurosurgery, Chiba Hokuso Hospital, Nippon Medical School, 1715, Kamagari, Inzai-city, Chiba, Japan

Tel: +81-476-99-1111, Fax: +81-476-99-1906, E-mail: kyongson@nms.ac.jp 


\section{Introduction}

Untreated neuropathic pain due to lumbar spine disease affects the activities of daily living and thus quality of life (QOL). The $\alpha 2 \delta$ subunits affect calcium channel trafficking and thus play an important role in peripheral neuropathic pain [1], with the $\alpha 2 \delta$-1 subunit in the central nervous system (CNS) being the main target for treating neuropathic pain and the $\alpha 2 \delta-2$ subunit involved in somnolence $[1,2]$. While pregabalin and gabapentin, nonselective ligands at the $\alpha 2 \delta-1$ and $\alpha 2 \delta-2$ subunits, effectively address peripheral neuropathic pain, their elicitation of adverse effects limits their efficacy [3].

Mirogabalin is a novel selective ligand for the $\alpha 2 \delta$ subunits of voltage-gated $\mathrm{Ca}$ channels [1,2,4-6]. Its binding affinities for the $\alpha 2 \delta-1$ and $\alpha 2 \delta-2$ subunits were greater than of pregabalin [1] and its dissociation rates are slower for $\alpha 2 \delta-1$ than $\alpha 2 \delta-2$. Pregabalin, on the other hand, dissociates the subunits simultaneously [1]. These findings suggest that mirogabalin may produce more potent and longer-lasting analgesia with fewer adverse CNS effects [1].

Phase 2 and 3 mirogabalin studies showed its efficacy for reducing pain and associated sleep dysfunction in patients with diabetic peripheral neuropathic pain and postherpetic neuralgia $[2,4,6-8]$. In a rat model, mirogabalin improved the sciatic nerve pain elicited by partial sciatic nerve ligation [1]; however, its effectiveness in patients with peripheral neuropathic pain due to lumbar spine disease remained to be evaluated. Results obtained from the mirogabalin treatment of patients with peripheral neuropathic pain due to lumbar spine disease were reported.

\section{Materials and Methods}

Our study was approved by the Research Ethics Committee of Nippon Medical School Chiba Hokusoh Hospital (IRB approval no., 775). All procedures were in accordance with the ethical standards of the institutional and national research committee and the revised Declaration of Helsinki. Our study is a retrospective study, and informed consent was obtained by opt-out under Institutional Review Board approval.

\section{Patients}

Included in this retrospective study were consecutive patients treated with mirogabalin for lower extremity radiculopathy due to lumbar spinal canal stenosis (LSS) or lumbar disc herniation (LDH). Five institutions participated in this study, namely, Nippon Medical School Chiba Hokusoh Hospital, Kushiro Rosai Hospital, Chiba Shintoshi Rurban Clinic, Katsutadai Hospital, and Tokyo Hikifune Hospital. Spinal surgeons made the diagnosis of LSS or LDH based on subjectively identified symptoms and magnetic resonance imaging (MRI) and neurological findings. The subjectively reported pain and/or numbness in the affected dermatome corresponded with MRI findings. The symptoms were numbness or tingling in the buttocks and lower extremities and motor weakness. In this study, severe neuropathic pain in the lower extremities with a Numerical Rating Scale (NRS) between 4 and 8 was also included [4].

Excluded were patients whose subjective pain assessment was hampered by psychiatric disorders, dementia, diabetes mellitus, and complex regional pain syndrome, as well as patients with fresh vertebral fractures, spinal tumors, peripheral vascular disorders, renal dysfunction (creatinine clearance $<60 \mathrm{~mL} / \mathrm{min}$ ), malignant tumors diagnosed 2 years earlier, and those with a history of lumbar surgery or nerve block treatment within 2 months before mirogabalin treatment. Candidates for surgery due to lumbar disorders eliciting paralysis or bladder dysfunction were also excluded.

Between April and December 2019, we started with 60 consecutive patients with peripheral neuropathic pain due to lumbar spine disease on mirogabalin. There were 43 women and 17 men ranging in age from 25 to 87 years (mean age, 67.6 \pm 12.4 years); 48 presented with LSS and 12 with LDH. However, mirogabalin treatment was stopped in eight patients before the end of the 8-week study period, due to insufficient pain reduction: two underwent nerve blockage, two lumbar surgery, two stopped coming to the outpatient clinic, one suffered a vertebral fracture, or one reported excessive weight gain. The remaining 52 patients were divided into group $1(\mathrm{n}=17$, leg symptoms lasting fewer than 3 months) and group $2(n=35$, leg symptoms for more than 3 months).

\section{Mirogabalin treatment}

In the first week, the patients received doses of mirogabalin at $10 \mathrm{mg} /$ day (5 mg twice daily), which was increased by 5 to $10 \mathrm{mg}$ every week as necessary for pain control. 
The maximum dose was $30 \mathrm{mg} /$ day and the patients were allowed to reduce the dose if they experienced adverse effects. When pain control was inadequate, they also received nonsteroidal anti-inflammatory drugs and acetaminophen; no other drugs, such as pregabalin, antiepileptics, serotonin and norepinephrine reuptake inhibitors, hypnotics, anxiolytics, or opioids, were administered during the study period [4]. We evaluated the treatment outcome in 52 patients who had continued mirogabalin treatment for 8 consecutive weeks.

\section{Evaluation of the treatment effects}

The NRS for leg pain or numbness and for low back pain (LBP; $0=$ no pain, $10=$ worst pain) was recorded before and 4 and 8 weeks after the start of treatment [9]. RolandMorris Disability Questionnaire (RDQ) scores and the NRS for sleep $(0=$ no sleep, $10=$ excellent sleep) obtained before and 8 weeks after the start of treatment were compared. The health-related QOL, based on the EuroQol five-dimension five-level scale (EQ-5D-5L) scale, was also compared before and 8 weeks after the start of mirogabalin treatment. The scale ranged from 0 to 1 , with 1 indicating perfect health. Drug side effects were also evaluated.

\section{Statistical analysis}

Statistical analysis was performed using IBM SPSS for Windows ver. 25.0 (IBM Corp., Armonk, NY, USA). The scores before and 4 and 8 weeks after the start of treatment were compared using the Friedman non-parametric test. Comparison of the scores recorded before and after 8 weeks of treatment were made using the Wilcoxon signedrank test. Gender and intergroup comparisons were subjected to the Mann-Whitney $U$-test. The Spearman correlation coefficient was used to verify the correlation between leg symptom abatement and sleep disturbance. Values are expressed as the mean \pm standard deviation. Differences of $p<0.05$ were considered statistically significant.

\section{Results}

\section{Evaluation after 8-week mirogabalin treatment}

Of the 60 patients, 52 were evaluable (LSS: $n=43$; LDH: $\mathrm{n}=9$ ), comprised of 35 women and 17 men (age range, 25-87 years; mean age, $67.6 \pm 12.4$ years). Their symptom

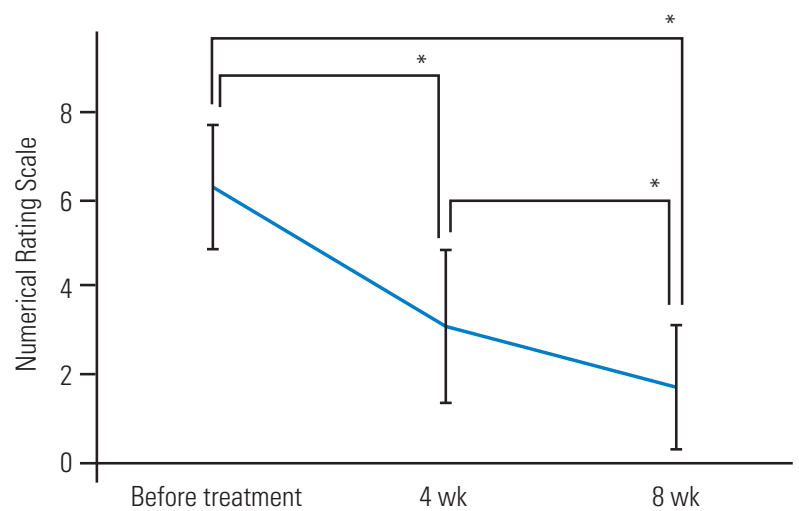

Fig. 1. Lower extremity symptoms improved significantly after 4- and 8-week mirogabalin treatment $\left({ }^{*} p<0.05\right)$.

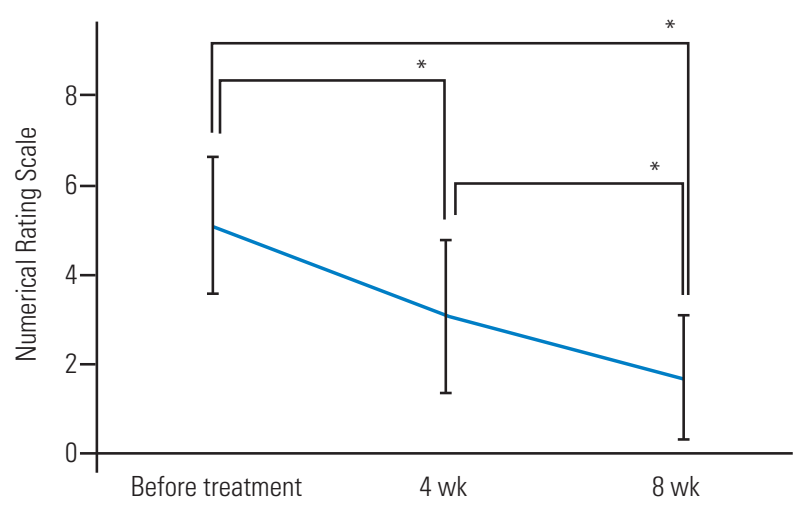

Fig. 2. Low back pain improved significantly after 4- and 8-week mirogabalin treatment $(* 0<0.05)$.

duration ranged from 1 to 468 months (mean, $34.9 \pm 78.9$ months). The mirogabalin dose ranged from 5 to $10 \mathrm{mg}$ (mean, $9.7 \pm 1.2 \mathrm{mg}$ ) in the first 2-week period, from 5 to $20 \mathrm{mg}$ (mean, $14.4 \pm 5.2 \mathrm{mg}$ ) in the second 2-week period, from 5 to $30 \mathrm{mg}$ (mean, $16.3 \pm 7.2 \mathrm{mg}$ ) in the third 2-week period, and from 5 to $30 \mathrm{mg}$ (mean, $17.0 \pm 7.8 \mathrm{mg}$ ) in the last 2-week period.

\section{Symptom alleviation}

Before treatment, the mean NRS for leg symptoms was 6.4 \pm 1.4. As shown in Fig. 1, with mirogabalin administration, it significantly improved after 4 weeks $(p<0.05)$ and decreased to $1.8 \pm 1.4$ after 8 weeks $(p<0.05)$. Of the 52 patients, 45 reported LBP before treatment; their mean NRS was $5.2 \pm 1.5$, and their mean RDQ score was 7.5 \pm 4.3 . Mirogabalin improved their LBP and the degree of improvement was significant at 4 weeks of treatment (3.2 \pm 1.6 , $p<0.05)$; their mean NRS was $1.8 \pm 1.4$ and their mean RDQ score was $2.4 \pm 3.0$ at the final visit $(p<0.05)$ (Fig. 2). 
Sleep disturbance was reported by 26 of the 52 patients. Their mean NRS for sleep was $5.9 \pm 1.9$ before treatment and $9.7 \pm 0.7$ at the last visit $(p<0.05)$. There was a weak correlation between leg symptom reduction and decreased sleep disturbance $(p=0.391, p<0.05)$. The EQ-5D-5L score for the QOL improved significantly $(0.54 \pm 0.08$ before mirogabalin and $0.75 \pm 0.15$ after mirogabalin, $p<0.05)$.

\section{Gender-based effects of mirogabalin}

There was no significant difference in the age of the male and female patients. After 8 weeks of mirogabalin treatment, the degree of improvement in their leg symptoms and QOL was also not significantly different (Table 1).

\section{Comparison of patients with leg symptoms lasting less or more than 3 months}

The 52 evaluable patients were divided based on the length of leg symptom duration. In group $1(n=17)$, it was less than 3 months; in group $2(n=35)$, it exceeded 3 months [10-12]. Before mirogabalin treatment, group 1 reported significantly worse symptoms and a significantly lower QOL than group $2(p<0.05)$. After 8 weeks of treatment, the improvement in leg symptoms and in the QOL was significantly better in group 1 than group $2(p<0.05)$ (Table 2).

\section{Adverse effects}

Excluded from our outcome evaluation were eight of 60 patients whose treatment was stopped early due to insufficient pain reduction; two underwent nerve blockage, two lumbar surgery, two stopped coming to the outpatient clinic, and one each suffered a vertebral fracture or reported excessive weight gain. Of the 60 original patients, 17 (28.3\%) suffered adverse effects, which were mild in 16 patients and required treatment cessation due to excessive weight gain in one patient.

\section{Discussion}

\section{Mirogabalin, a novel $\alpha 2 \delta$ ligand}

Neuropathic pain affects the QOL. Worldwide, pregabalin and gabapentin, selective ligands for the $\alpha 2 \delta$ subunits of voltage-gated calcium channels, are the first-line drugs for its treatment $[13,14]$. They have also been administered to treat leg symptoms associated with lumbar disease $[9,15,16]$. However, their side effects, for example, dizziness and somnolence, prohibit their prolonged and highdose administration $[3,9,17]$. The $\alpha 2 \delta-1$ subunit contributes to the stimulation of analgesic effects, and the $\alpha 2 \delta$ 2 subunit to CNS side effects [1,2]. Consequently, ligand selectivity for $\alpha 2 \delta-1$ and $\alpha 2 \delta-2$ may produce different clinical outcomes.

Table 1. Gender difference in the treatment outcomes

\begin{tabular}{|c|c|c|c|c|c|c|c|}
\hline \multirow{2}{*}{ Gender } & \multirow{2}{*}{ Mean age (yr) } & \multirow{2}{*}{ Diagnosis } & \multicolumn{2}{|c|}{ Leg symptoms (NRS) } & \multicolumn{2}{|c|}{$00 \mathrm{~L}$} & \multirow{2}{*}{$p$-value } \\
\hline & & & Pre-treatment & 8 wk post-treatment & Pre-treatment & 8 wk post-treatment & \\
\hline Male (n=17) & 68.3 & 16 (LSS), 1 (LDH) & $6.6 \pm 1.2$ & $1.8 \pm 1.6$ & $0.54 \pm 0.08$ & $0.76 \pm 0.17$ & $<0.05$ \\
\hline Female $(n=35)$ & 67.2 & 27 (LSS), 8 (LDH) & $6.2 \pm 1.5$ & $1.7 \pm 1.3$ & $0.54 \pm 0.09$ & $0.74 \pm 0.15$ & $<0.05$ \\
\hline
\end{tabular}

Values are presented as mean \pm standard deviation, unless otherwise stated.

NRS, Numerical Rating Scale; 0OL, quality of life based on the EuroOol five-dimension five-level scale; LSS, lumbar spinal canal stenosis; LDH, lumbar disc herniation.

Table 2. Treatment outcomes in patients with group 1 or group $2^{\text {a) }}$

\begin{tabular}{|c|c|c|c|c|c|c|}
\hline Group & Mean age (yr) & Gender & Diagnosis & Leg symptoms (NRS) & $\mathrm{QOL}^{\mathrm{a})}$ & $p$-value \\
\hline $1(n=17)$ & 66.6 & $\mathrm{M8}, \mathrm{Fg}$ & 13 (LSS), 4 (LDH) & $7.2 \pm 1.2$ to $1.3 \pm 1.0$ & $0.49 \pm 0.07$ to $0.79 \pm 0.15$ & $<0.05$ \\
\hline $2(n=35)$ & 68.2 & M9, F26 & 30 (LSS), 5 (LDH) & $6.0 \pm 1.4$ to $2.0 \pm 1.6$ & $0.57 \pm 0.08$ to $0.73 \pm 0.14$ & $<0.05$ \\
\hline
\end{tabular}

Values are presented as mean \pm standard deviation, unless otherwise stated.

NRS, Numerical Rating Scale; 0OL, quality of life based on the EuroQol 5-dimension 5-level scale; M, male; F, female; LSS, lumbar spinal canal stenosis; LDH, lumbar disc herniation.

a) The interval from symptom onset to the start of treatment was less than 3 months in group 1 and exceeded 3 months in group 2 . 
Mirogabalin, a novel ligand for the $\alpha 2 \delta$ subunits of voltage-gated calcium channels, can be used to treat neuropathic pain $[1,5]$. As it has potent selective binding affinities for $a 2 \delta$ subunits, and because its dissociation rates are slower for $\alpha 2 \delta$-1 than $\alpha 2 \delta$-2, it may produce long-lasting analgesic effects in patients with neuropathic pain and fewer and milder CNS side effects [1]. Exposure-response modeling in diabetics with peripheral neuropathic pain suggested that mirogabalin was superior to pregabalin $[2,5]$. Rat models of neuropathic pain elicited by partial sciatic nerve ligation or streptozotocin-induced diabetes revealed that mirogabalin produced more potent and longer-lasting analgesic effects than pregabalin; its safety indices were also superior to pregabalin [1].

\section{Effects of mirogabalin on leg symptoms}

Mirogabalin was effective in patients with diabetic peripheral neuropathic pain and postherpetic neuralgia $[2,4,6$ 8]. However, its effectiveness in patients with non-chronic phase neuropathic pain remained unknown. We report its usefulness in the presence of chronic and non-chronic neuropathic leg symptoms in patients with lumbar spinal disease. According to Vinik et al. [2], the median time to significant pain reduction was within 30 days for all mirogabalin doses they administered. We observed significant reduction of leg symptoms 4 weeks after the start of mirogabalin administration; its effect was relatively rapid. In Asians, the efficacy of mirogabalin was dose-dependent; Baba et al. [4] reported that the administered dose for treating diabetic peripheral neuropathic pain decreased after week 1 and fell further in the course of 14 weeks, suggesting a cumulative effect of mirogabalin.

Although we allowed our patients to control the dose, it did not change significantly throughout the 8-week study period. While their leg symptoms improved after each 4-week treatment, there was a discrepancy between the drug effect and the drug dose. Mirogabalin was more effective in group 1 than group 2; however, pain control was insufficient in four of the original 60 patients and two each required surgical or nerve block intervention. This indicates that the effectiveness of monotherapy may be limited.

\section{Effects of mirogabalin on low back pain}

In our patients, mirogabalin significantly reduced LBP associated with leg symptoms due to lumbar spine disease.
In some patients, LBP was neuropathic pain; pregabalin alleviated both $[17,18]$. It was also useful for perioperative LBP control after lumbar discectomy [15]. Neuropathic pain due to nerve root involvement is a common cause of LBP $[19,20]$, and more than one-third of LBP is accompanied by neuropathic pain [21].

Elsewhere, we reported that superior/middle cluneal nerve entrapment around the iliac crest can elicit LBP; the symptoms are similar to LBP due to coexisting lumbar disease $[22,23]$ whose clinical and pathological features are those of peripheral nerve neuropathy [23]. Neuropathic pain from these diseases may be alleviated by mirogabalin. Our diagnosis was based on the effectiveness of cluneal nerve blocks. We did not deliver blocks to patients whose outcomes after mirogabalin treatment were evaluated.

\section{Effect of mirogabalin on sleep disturbance and quality of life}

Sleep disturbance and anxiety affect the QOL; they are often patient-reported effects of neuropathic pain $[24,25]$. There is a relationship between pain and sleep, as neuropathic pain can directly interfere with sleep. Poor sleep can increase pain sensitivity and lower the pain threshold $[6,17]$. Sleep disturbance may impair descending pain inhibition pathways that play an important role in pain control; good sleep may help to reduce pain $[26,27]$.

Pregabalin significantly improved patient-reported sleep disturbance across multiple conditions including neuropathic pain. It exerts indirect analgesic effects and direct effects on sleep $[17,28]$. Mirogabalin significantly reduced sleep interference due to diabetic peripheral neuropathic pain and postherpetic neuralgia $[4,8]$. We found that it also significantly reduced sleep disturbance in patients with neuropathic pain due to lumbar diseases. We think that the drug relieves anxiety and neuropathic pain and that the improvement of sleep disturbance is its direct effect; pain reduction may be a secondary effect $[6,29]$.

As pregabalin alleviates not only neuropathic pain but also associated symptoms such as sleep disturbance, anxiety, and mood disorders, it may help to improve the overall patient health $[9,16,17]$. Vinik et al. [25] suggested that improvements in patient function and health produced by pregabalin derive from a combination of effects, that is, analgesic effects and a reduction in sleep interference. The QOL of our patients, based on the EQ-5D-5L, improved after 8 weeks of mirogabalin treatment. We suggest that 
the drug exerts a combination of effects, that is, analgesic effects on leg symptoms and LBP and a reduction in sleep interference.

\section{Adverse effects}

Although pregabalin was safe and well tolerated, the incidence of adverse effects ranged from $12.5 \%$ to approximately $25.5 \%[9,17]$. The most common complaints were somnolence and dizziness; nausea, edema, and weight gain were also reported $[9,17,18]$. As mirogabalin binds longer to the $\alpha 2 \delta$-1 subunit, which plays an important role in its analgesic effect, than the $\alpha 2 \delta-2$ subunit, which is related to CNS side effects, a low incidence of adverse effects can be expected [1]. Others reported that a long-term, flexible mirogabalin dosing regimen was safe and effective $[1,4,7]$; the adverse effects were somnolence, dizziness, peripheral edema, and weight gain $[1,2,4,7,8]$. According to Vinik et al. [2], in 277 mirogabalin-treated patients, the most frequent adverse effects were mild to moderate dizziness (9.4\%), somnolence (6.1\%), and headache (6.1\%).

Our patients reported mild somnolence (15.0\%), mild dizziness (6.7\%), edema and weight gain (6.7\%), and dry mouth (3.3\%). A single patient experienced excessive weight gain, possibly due to our nonrigid dosing regimen, and discontinued taking the drug. In Japan, the maximum allowable daily mirogabalin dose is $30 \mathrm{mg}$. Others reported the incidence of adverse effect increased with increasing doses $[2,4,8]$. In our series, dosing was flexible; the mean final dose was $17.0 \mathrm{mg}$ (range, 5 to $30 \mathrm{mg}$ ). Patient control over the mirogabalin dose may help to reduce the rate of adverse effects. Of the 60 patients, only one stopped taking the drug due to adverse effects, and 16 patients reported mild adverse effects.

Chen et al. [30] reported that, besides weight gain, hair loss is a common cosmetic side effect of antiepileptic drugs, including pregabalin; the underlying mechanisms remain unknown. It was observed in 24 of 270 patients treated with valproic acid (8.9\%) and in one of $143 \mathrm{pa}$ tients $(0.7 \%)$ who took pregabalin. At this time, hair loss has not been reported as an adverse effect of mirogabalin $[1,2,4,7,8]$, and we did not observe it in our series. However, as ours was a retrospective study, we cannot comment on this issue and it remains a topic requiring further analyses.

\section{Limitations}

Our study was retrospective and the study population was small. As we had no controls, we cannot deny a placebo effect. Prospective, randomized control studies using a large patient population are needed. Lastly, our comparatively short follow-up period ( 8 versus 52 weeks) may have affected our documented incidence of adverse effects [7]. However, Baba et al. [7] who followed their mirogabalin-treated patients with diabetic peripheral neuropathic pain for 52 weeks also reported a decrease in pain after 8-week treatment; the pain-reducing effect persisted for 52 weeks. As we evaluated sleep disturbance and QOL issues only before and after 8 weeks of treatment, we cannot comment on the exact timing of their improvement in the 8 -week time course.

\section{Conclusions}

We first report the beneficial effects of mirogabalin in patients with neuropathic pain due to lumbar spine disease. Its effects were more pronounced in patients whose pain had lasted for less than 3 months than in those whose symptom duration was longer than 3 months. Based on our findings, we think that mirogabalin is useful for the treatment of not only leg symptoms but also of LBP and sleep disturbance associated with lumbar spine disease.

\section{Conflict of Interest}

All authors who are members of the Japan Neurosurgical Society (JNS) have completed the Self-reported COI Disclosure Statement Forms available at the website for JNS members. No potential conflict of interest relevant to this article was reported.

\section{References}

1. Domon Y, Arakawa N, Inoue T, et al. Binding characteristics and analgesic effects of mirogabalin, a novel ligand for the $\alpha 2 \delta$ subunit of voltage-gated calcium channels. J Pharmacol Exp Ther 2018;365:573-82.

2. Vinik A, Rosenstock J, Sharma U, et al. Efficacy and safety of mirogabalin (DS-5565) for the treatment of diabetic peripheral neuropathic pain: a randomized, double-blind, placebo- and active comparatorcontrolled, adaptive proof-of-concept phase 2 study. 
Diabetes Care 2014;37:3253-61.

3. Yang M, Qian C, Liu Y. Suboptimal Treatment of diabetic peripheral neuropathic pain in the United States. Pain Med 2015;16:2075-83.

4. Baba M, Matsui N, Kuroha M, Wasaki Y, Ohwada S. Mirogabalin for the treatment of diabetic peripheral neuropathic pain: a randomized, double-blind, placebo-controlled phase III study in Asian patients. J Diabetes Investig 2019;10:1299-306.

5. Hutmacher MM, Frame B, Miller R, Truitt K, Merante D. Exposure-response modeling of average daily pain score, and dizziness and somnolence, for mirogabalin (DS-5565) in patients with diabetic peripheral neuropathic pain. J Clin Pharmacol 2016;56:6777.

6. Merante D, Rosenstock J, Sharma U, et al. Efficacy of mirogabalin (DS-5565) on patient-reported pain and sleep interference in patients with diabetic neuropathic pain: secondary outcomes of a phase II proofof-concept study. Pain Med 2017;18:2198-207.

7. Baba M, Matsui N, Kuroha M, Wasaki Y, Ohwada S. Long-term safety and efficacy of mirogabalin in Asian patients with diabetic peripheral neuropathic pain. J Diabetes Investig 2020;11:693-8.

8. Kato J, Matsui N, Kakehi Y, Murayama E, Ohwada S, Sugihara M. Mirogabalin for the management of postherpetic neuralgia: a randomized, double-blind, placebo-controlled phase 3 study in Asian patients. Pain 2019;160:1175-85.

9. Orita S, Yamashita M, Eguchi Y, et al. Pregabalin for refractory radicular leg pain due to lumbar spinal stenosis: a preliminary prospective study. Pain Res Manag 2016;2016:5079675.

10. Chou R, Qaseem A, Snow V, et al. Diagnosis and treatment of low back pain: a joint clinical practice guideline from the American College of Physicians and the American Pain Society. Ann Intern Med 2007;147:478-91.

11. Buchner M, Neubauer E, Zahlten-Hinguranage A, Schiltenwolf $M$. The influence of the grade of chronicity on the outcome of multidisciplinary therapy for chronic low back pain. Spine (Phila Pa 1976) 2007;32:3060-6.

12. Karjalainen K, Malmivaara A, Mutanen P, Roine R, Hurri H, Pohjolainen T. Mini-intervention for subacute low back pain: two-year follow-up and modifiers of effectiveness. Spine (Phila Pa 1976)
2004;29:1069-76.

13. Moulin DE, Clark AJ, Gilron I, et al. Pharmacological management of chronic neuropathic pain: consensus statement and guidelines from the Canadian Pain Society. Pain Res Manag 2007;12:13-21.

14. Ogawa S, Satoh J, Arakawa A, Yoshiyama T, Suzuki M. Pregabalin treatment for peripheral neuropathic pain: a review of safety data from randomized controlled trials conducted in Japan and in the West. Drug Saf 2012;35:793-806.

15. Burke SM, Shorten GD. Perioperative pregabalin improves pain and functional outcomes 3 months after lumbar discectomy. Anesth Analg 2010;110:1180-5.

16. Saldana MT, Navarro A, Perez C, Masramon X, Rejas J. Patient-reported-outcomes in subjects with painful lumbar or cervical radiculopathy treated with pregabalin: evidence from medical practice in primary care settings. Rheumatol Int 2010;30:1005-15.

17. Taguchi T, Igarashi A, Watt S, et al. Effectiveness of pregabalin for the treatment of chronic low back pain with accompanying lower limb pain (neuropathic component): a non-interventional study in Japan. J Pain Res 2015;8:487-97.

18. Freynhagen R, Baron R, Gockel U, Tolle TR. painDETECT: a new screening questionnaire to identify neuropathic components in patients with back pain. Curr Med Res Opin 2006;22:1911-20.

19. Dionne CE, Dunn KM, Croft PR, et al. A consensus approach toward the standardization of back pain definitions for use in prevalence studies. Spine (Phila Pa 1976) 2008;33:95-103.

20. Webb R, Brammah T, Lunt M, Urwin M, Allison T, Symmons D. Prevalence and predictors of intense, chronic, and disabling neck and back pain in the UK general population. Spine (Phila Pa 1976) 2003;28:1195-202.

21. Fishbain DA, Cole B, Lewis JE, Gao J. What is the evidence that neuropathic pain is present in chronic low back pain and soft tissue syndromes?: an evidence-based structured review. Pain Med 2014;15:415.

22. Isu T, Kim K, Morimoto D, Iwamoto N. Superior and middle cluneal nerve entrapment as a cause of low back pain. Neurospine 2018;15:25-32.

23. Kim K, Shimizu J, Isu T, et al. Low back pain due to superior cluneal nerve entrapment: a clinicopathologic study. Muscle Nerve 2018;57:777-83. 
24. Schaefer C, Mann R, Sadosky A, et al. Burden of illness associated with peripheral and central neuropathic pain among adults seeking treatment in the United States: a patient-centered evaluation. Pain Med 2014;15:2105-19.

25. Vinik A, Emir B, Cheung R, Whalen E. Relationship between pain relief and improvements in patient function/quality of life in patients with painful diabetic peripheral neuropathy or postherpetic neuralgia treated with pregabalin. Clin Ther 2013;35:61223.

26. Choy EH. The role of sleep in pain and fibromyalgia. Nat Rev Rheumatol 2015;11:513-20.

27. Davies KA, Macfarlane GJ, Nicholl BI, et al. Restorative sleep predicts the resolution of chronic widespread pain: results from the EPIFUND study. Rheumatology (Oxford) 2008;47:1809-13.
28. Vinik A, Emir B, Parsons B, Cheung R. Prediction of pregabalin-mediated pain response by severity of sleep disturbance in patients with painful diabetic neuropathy and post-herpetic neuralgia. Pain Med 2014;15:661-70.

29. Murasawa H, Kobayashi H, Saeki K, Kitano Y. Anxiolytic effects of the novel $\alpha 2 \delta$ ligand mirogabalin in a rat model of chronic constriction injury, an experimental model of neuropathic pain. Psychopharmacology (Berl) 2020;237:189-97.

30. Chen B, Choi H, Hirsch LJ, et al. Cosmetic side effects of antiepileptic drugs in adults with epilepsy. Epilepsy Behav 2015;42:129-37. 